\title{
UNE THÈSE FÉMINISTE SUR L'ORIGINE DE L'OBÉSITÉ
}

\author{
Susie Orbach : Fat is a feminist issue. Berkley, Books, New York 1978. \\ Traduction française : Maigrir sans obsession. Édition "Le Jour ", Montréal, 1984, 233 p. (traduit de \\ l'américain par Sylvie Dupont).
}

Ce livre écrit par une psychothérapeute nord-américaine, apporte un éclairage original sur la question de l'obésité de femmes (exclusivement) et sur leur comportement associé de "mangeuses compulsives ", à partir d'un travail fait aux USA, au Canada et en Angleterre, auprès de femmes blanches de 17 à 65 ans.

La thèse centrale de l'ouvrage est une thèse féministe : l'obésité (et, à des degrés divers, les problèmes de "poids" si fréquents chez les femmes contemporaines) serait pour une grande partie une réponse à la pression exercée par la société patriarcale sur les images-modèles imposées aux femmes. Au titre de cette réponse, l'obésité est en fait inconsciemment désirée par les femmes et non pas subie, comme les apparences (et les sujets eux-mêmes) peuvent le faire croire.

Ce "choix" de la grosseur est à comprendre en parallèle à la peur que la minceur suscite chez bien des femmes. La minceur, si prônée par tout l'environnement social (mode, médias, corps médical) est en fait associée pour les mangeuses compulsives à un double risque.

1) Le risque de se conformer précisément aux attentes sociales extérieures à elles-mêmes, attentes des autres, que toutes les femmes ont appris à privilégier sur les leurs propres au cours de leur éducation. Ces attentes sont de plus contradictoires : le corps mince de la femme, par exemple, est d'abord "l'objet " sexuel désiré par les hommes; mais la femme comme davantage égoïste et narcissique que la femme ronde, image maternelle généreuse et rassurante, autre attente sociale. Ces deux modèles sont imposés aux femmes indépendamment de ce qu'elles se sentent et se savent être comme personnes, qui pensent et qui agissent, quelle que soit leur apparence physique.

Par l'obésité, les femmes contestent donc (consciemment ou inconsciemment) les stéréotypes féminins définis par notre culture : l'obésité est ici une rébellion.

2) Le risque d'être confrontée à son impuissance individuelle à modifier le statut de "seconde zone " qui est celui des femmes dans les sociétés patriarcales ; impuissance renforcée par la difficulté des femmes à dire " non ", ou à se mettre en colère (autres images socialement négatives) ainsi que par le message contradictoire que les femmes ont reçu de leurs mères (qui, elles-mêmes, le tiennent de leur mère, etc.) : " sois comme moi / essaie de t'en sortir mieux".

Ces conflits insolubles sont déplacés sur celui du poids et du rapport à la nourriture, conflit qui semble plus accessible et plus maniable à chaque femme isolément et qui, par ailleurs, rend visibles leurs difficultés et mobilise une forme d'aide sociale : l'obèse préoccupe son entourrage et intéresse médias et médecins, entre autres.

Dans quelles conditions ces analyses sont-elles communiquées aux femmes mangeuses compulsives? Quelles solutions envisager?
L'auteur propose que les femmes obèses constituent de petits groupes d'entraide de cinq à huit personnes, groupes réguliers à horaire fixe $(1 \mathrm{~h} 30$ à 2 h 30$)$. Ces groupes n'ont pas besoin d'animatrices, l'égalité de position des participantes leur permet d'échanger leurs expériences et favorise l'émergence de la dimension sociale de leurs difficultés : les similitudes de leurs situations favorisent la prise de conscience du fait que leur obésité a un rapport avec leur condition féminine et n'est pas exclusivement un problème individuel. Ceci leur permet par ailleurs de sortir d'une clandestinité propre aux différentes " toxicomanies".

Ces groupes permettent également que la recherche de solutions ne se vive pas dans une situation " infantilisante " ou dévalorisante pour les femmes, comme peuvent l'être celles instituées par des autorités médicales ou médiatiques.

Ces groupes explorent entre autres, les représentations liées à la " grosseur " et à la " minceur " des participantes, à partir d'exercices proposés dans cet ouvrage à titre d'exemples et qui peuvent être modulés ou enrichis par les femmes elles-mêmes*.

Le livre se termine sur une brève revue des recherches médicales en cours sur l'obésité, dans une perspective critique.

\section{Claire Rueff-Escoubès}

- De très nombreux cas sont présentés, avec des résultats positifs à long terme: le poids stable est celui dans lequel chaque femme "se sent bien", ce n'est jamais l'obésité, ce n'est pas nécessairement une minceur " slandard". 